\title{
Attosecond Time-Resolved Autoionization of Argon
}

\author{
He Wang, ${ }^{1}$ Michael Chini, ${ }^{1}$ Shouyuan Chen, ${ }^{1}$ Chang-Hua Zhang, ${ }^{1}$ Feng He, ${ }^{2,1}$ \\ Yan Cheng, ${ }^{1}$ Yi Wu, ${ }^{1}$ Uwe Thumm, ${ }^{1}$ and Zenghu Chang ${ }^{1,3, *}$ \\ ${ }^{1}$ J.R. Macdonald Laboratory, Department of Physics, Kansas State University, Manhattan, Kansas 66506, USA \\ ${ }^{2}$ Department of Physics, Shanghai Jiaotong University, Shanghai 200240, China \\ ${ }^{3}$ CREOL and Department of Physics, University of Central Florida, Orlando, Florida 32816, USA
}

(Received 4 June 2010; published 1 October 2010)

\begin{abstract}
Autoionization of argon atoms was studied experimentally by transient absorption spectroscopy with isolated attosecond pulses. The peak position, intensity, linewidth, and shape of the $3 s 3 p^{6} n p{ }^{1} P$ Fano resonance series (26.6-29.2 eV) were modified by intense few-cycle near infrared laser pulses, while the delay between the attosecond pulse and the laser pulse was changed by a few femtoseconds. Numerical simulations revealed that the experimentally observed splitting of the $3 s 3 p^{6} 4 p^{1} P$ line is caused by the coupling between two short-lived highly excited states in the strong laser field.
\end{abstract}

DOI: 10.1103/PhysRevLett.105.143002

PACS numbers: 32.70.Jz, 32.80.Zb, 78.47.J-

Bridging the gap between atomic physics and the complex systems that make up the world around us requires indepth study of electron correlation. While rotation and vibration of molecules can be studied by femtosecond lasers [1], observation of the electron-electron interaction requires attosecond time resolution [2]. One of the most interesting processes governed by electron-electron correlation is autoionization [3]. The Fano profile, which is the signature of the autoionization process, has widespread significance in many scientific disciplines [4-7]. For decades, spectral-domain measurements with synchrotron radiation have served as a window into the rich dynamics of autoionization [4]. However, the synchrotron pulse duration is too long ( $100 \mathrm{fs}$ to $100 \mathrm{ps}$ ) to time-resolve the Fano resonances since the autoionization lifetimes can be as short as a few femtoseconds.

Since the generation of the first isolated attosecond pulses in 2001 [8], it was theoretically proposed [9-13] and experimentally demonstrated [14] that time-resolved Fano profiles can be studied using the attosecond streaking technique. To date, most theoretical and experimental investigations of autoionization processes have scrutinized Fano profiles as a function of the photoelectron energy. However, made possible by significant recent progress in short-pulse laser technology [15], timeresolved transient XUV photoabsorption measurements have become feasible, which gives access to complimentary studies of atomic autoionization in the time regime $[16,17]$. Photoabsorption measurements typically have higher data collection efficiency and better energy resolution than what can be obtained by detecting photoelectrons. The setup is all-optical, much simpler than the attosecond streak camera. Here we demonstrate the first transient absorption experiment using isolated attosecond pulses to probe the autoionization of atoms and show that the autoionization process is strongly modified by an intense laser field.
Fano resonance profiles in the absorption spectrum are the result of interference between the direct ionization and the decay from an autoionizing state due to configuration interaction [3]. It is characterized by the resonance energy $E_{r}$, its width that is related to the lifetime of the autoionizing state by $\tau=\hbar / \Gamma$, and the $q$ parameter, which represents the ratio of amplitudes for direct transitions from the ground state to the autoionizing state and transitions to this state via the continuum state. This parameter determines the shape of the resonance. A diagram of the argon autoionizing states of interest is depicted in Fig. 1(a). The line widths of the $3 s 3 p^{6} 4 p$ and $3 s 3 p^{6} 5 p$ states are
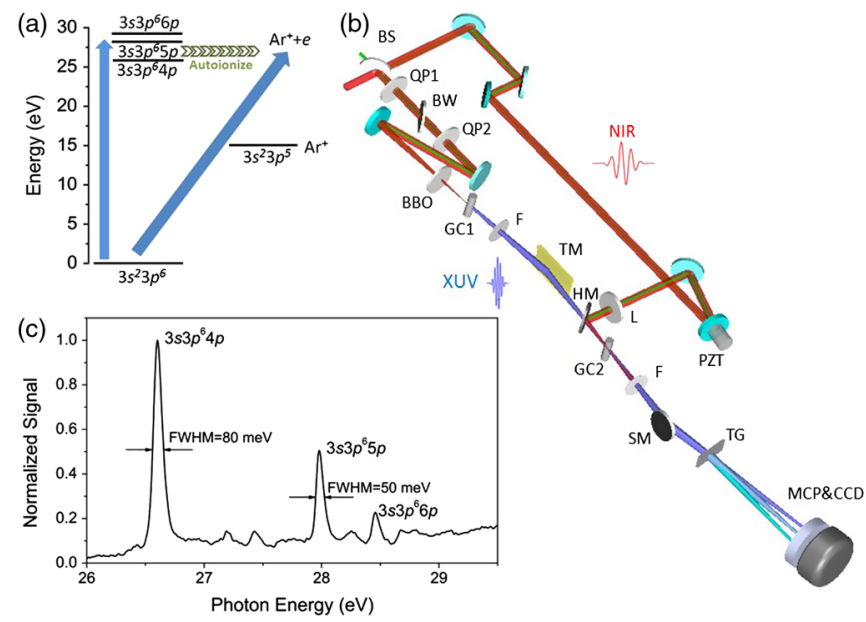

FIG. 1 (color online). (a) Energy diagram of the $3 s 3 p^{6} n p{ }^{1} P$ autoionizing states in argon. The continuum spectrum of the attosecond pulse covers the ${ }^{1} P$ series of states. (b) Attosecond transient absorption experimental setup. BS: beam splitter; QP1, BW, QP2, BBO: GDOG optics; GC1: HHG gas cell; F: aluminum filter; TM: toroidal mirror; GC2: absorption gas cell; L: lens; HM: hole mirror; SM: spherical mirror; TG: transmission grating. (c) Transmitted XUV spectrum indicating argon $3 s 3 p^{6} n p{ }^{1} P$ autoionizing states. The spectrometer resolution was $50 \mathrm{meV}$. 
80 and $28.2 \mathrm{meV}$, respectively [4], which correspond to lifetimes of 8.2 and $23.3 \mathrm{fs}$.

To control the autoionization process, a pump-probe scheme with a Mach-Zehnder configuration was used in the experiment as shown in Fig. 1(b). The carrier-envelope phase stabilized $1 \mathrm{~mJ}, 6$ to $8 \mathrm{fs}$ NIR pulses centered at $750 \mathrm{~nm}$ [18] were split into two parts. Half of the beam generated the isolated attosecond pulse using the generalized double optical gating (GDOG) [19] from argon gas, and the corresponding XUV supercontinuum spectrum covered the energy range between 20 and $40 \mathrm{eV}$. Measurements with an attosecond streak camera and reconstruction by the FROG-CRAB method confirmed the pulse duration to be $\sim 140$ as [19]. The attosecond XUV pulse passed through a $300 \mathrm{~nm} \mathrm{Al} \mathrm{foil} \mathrm{and} \mathrm{was} \mathrm{focused} \mathrm{by} \mathrm{a}$ toroidal mirror $\left(f=250 \mathrm{~mm}, 9.6^{\circ}\right.$ grazing incidence angle) to a second glass gas cell with a $1 \mathrm{~mm}$ inner diameter and $\sim 30 \mu \mathrm{m}$ diameter hole on each side filled with 25 torr of argon gas where more than $80 \%$ of the XUV was absorbed.

Meanwhile, the other half of the NIR beam was recombined collinearly with the attosecond pulse at the second gas cell by a hole-drilled mirror which reflected a portion of the NIR and allowed the XUV to pass. A lens with $f=$ $400 \mathrm{~mm}$ was used to focus the NIR to the second gas cell (Rayleigh range $\sim 3 \mathrm{~mm}$ ). The delay between the NIR and XUV pulses was introduced by a piezo-electric transducer. A cw green laser was copropagated in both arms of the interferometer to stabilize and control the delay between the NIR and XUV pulses [20].

The XUV pulse was transmitted through the second cell and refocused by a spherical mirror at a grazing incidence angle of $2^{\circ}$, and its spectrum was dispersed by a transmission grating (2000 lines $/ \mathrm{mm}$ ) [21] on the MCPphosphor and $\mathrm{CCD}$ image recorder. A second $\mathrm{Al}$ filter was installed before the XUV spectrometer to block the scattered NIR light. The spectrometer resolution was estimated to be $50 \mathrm{meV}$ by using the $3 s 3 p^{6} 5 p$ peak $(\Gamma=$ $28.2 \mathrm{meV}$ ) as a reference, and its energy scale was calibrated by the position of the $3 s 3 p^{6} 4 p\left(E_{r}=26.6 \mathrm{eV}\right)$, $3 s 3 p^{6} 5 p(28.0 \mathrm{eV})$, and $3 s 3 p^{6} 6 p(28.5 \mathrm{eV})$ resonance peaks as shown in Fig. 1(c).

Figure 2(a) shows the transmitted XUV spectrum for different delays between the XUV and NIR pulses with a peak NIR intensity of $\sim 5 \times 10^{11} \mathrm{~W} / \mathrm{cm}^{2}$. In the plot, negative delays correspond to XUV pulses arriving on the argon target before the NIR pulses. Three resonance peaks $3 s 3 p^{6} 4 p, 3 s 3 p^{6} 5 p$, and $3 s 3 p^{6} 6 p$ are labeled. Weaker lines lying between the resonance peaks are artifacts of the transmission grating spectrometer. As the time delay approaches zero, the NIR pulse gradually shifts the positions of the resonance peaks to higher energy. The shifted resonance peaks are also broadened and weakened. Surprisingly, the effects of the laser are more apparent on the lower-lying autoionizing states.

In Fig. 2(c), the transmitted XUV signal at the energy of the unperturbed $3 s 3 p^{6} 4 p(26.6 \mathrm{eV})$ and $3 s 3 p^{6} 5 p(28.0 \mathrm{eV})$
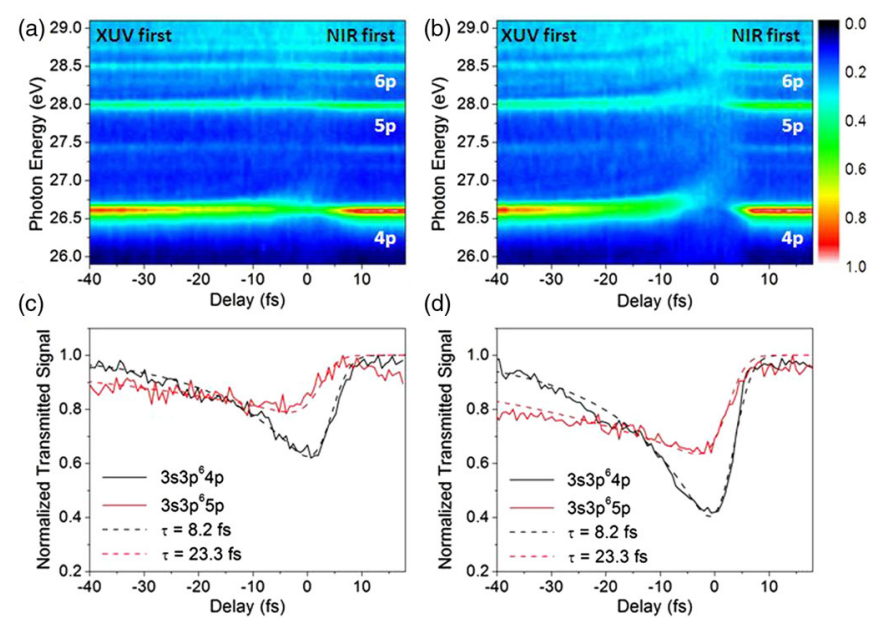

FIG. 2 (color online). Transmitted attosecond XUV spectra of argon in a strong NIR laser field with a peak intensity of (a) $5 \times 10^{11} \mathrm{~W} / \mathrm{cm}^{2}$ and (b) $10^{12} \mathrm{~W} / \mathrm{cm}^{2}$. Negative delays correspond to the attosecond pulse arriving on the target before the NIR laser pulse. The resonance peaks are shifted, broadened, and weakened when the two pulses overlap. (c),(d) Transmitted signal (solid) near the $3 s 3 p^{6} 4 p$ and $3 s 3 p^{6} 5 p$ states for $5 \times$ $10^{11} \mathrm{~W} / \mathrm{cm}^{2}$ and $10^{12} \mathrm{~W} / \mathrm{cm}^{2}$, respectively, and calculated exponential decay convoluted with 4.5 fs Gaussian for best fit (dashed).

peaks is plotted as a function of the delay. When the XUV and NIR overlap temporally, the transmitted signals are minimized. The recovery of the signal is substantially faster when the delay is positive. The asymmetric weakening of the signal with respect to delay can be fit very well using a cross-correlation of an exponential function with the autoionization state lifetimes and a Gaussian laser pulse [4].

To study the effect of the NIR laser intensity on the target, the delay-dependent transmitted XUV spectrum was measured again under the same conditions but with a higher NIR laser peak intensity of $\sim 10^{12} \mathrm{~W} / \mathrm{cm}$, as is shown in Fig. 2(b). The $3 s 3 p^{6} 5 p$ and $3 s 3 p^{6} 6 p$ states again primarily exhibit an energy shift as well as broadening and weakening of the resonances, but the effects were enhanced compared with the results for the lower intensity in Fig. 2(a). Most interestingly, $3 s 3 p^{6} 4 p$ exhibits a dramatic splitting which is asymmetric with respect to zero delay. The upper branch extends nearly to the neighboring $3 s 3 p^{6} 5 p$ peak, whereas the lower branch remains near the unperturbed energy.

The observed phenomena suggest a dynamic control over the autoionizing states by the NIR laser, which can be understood by considering both nonresonant coupling of the autoionizing states to continuum states and resonant coupling to other autoionizing states induced by the NIR laser $[9,10,22]$. In our experiment, $3 s 3 p^{6} n p{ }^{1} P$ autoionizing states in argon are first populated by the single attosecond XUV pulse. If no NIR field is present, those states will decay exponentially to the $\mathrm{Ar}^{+}\left(3 s^{2} 3 p^{5} \varepsilon l\right)$ continuum due to configuration interaction. When those states are further dressed by a strong laser, additional couplings 
between the autoionizing states and $\operatorname{Ar}^{*+}\left(3 s 3 p^{6} \varepsilon l\right)$ continuum states by single- or multiphoton ionization (1.65 eV photon energy for $750 \mathrm{~nm}$ central wavelength) tend to speed up the decay process and therefore broaden the autoionization peak as shown in Fig. 3(a) and 3(b). Similarly to the ac Stark shift in Rydberg atoms, extra couplings to the continuum states caused by the NIR laser shift the central energies of the autoionization peaks [22].

When resonant coupling to other autoionizing states is also possible, such strong coupling may take over as the dominant mechanism for controlling the autoionization process. Since the $3 s 3 p^{6} 4 p(26.6 \mathrm{eV})$ state is more deeply bound than the $3 s 3 p^{6} 5 p$ and $3 s 3 p^{6} 6 p$ states with respect to the $\mathrm{Ar}^{*+}\left(3 s 3 p^{6} \varepsilon l\right)$ continuum, coupling only to the $\mathrm{Ar}^{*+}$ continuum indicates that the $3 s 3 p^{6} 4 p$ should be less sensitive to the NIR laser. However, the experimental data show an even stronger shift and broadening of the $3 s 3 p^{6} 4 p$ autoionization peak, as well as the asymmetric splitting at high laser intensity, which can be explained by the resonant coupling $[9,10]$. Specifically, the $3 s 3 p^{6} n d$ states of argon, which lie in the same energy range as the $3 s 3 p^{6} n{ }^{1} P$ manifold of states, cannot be accessed by the absorption of one XUV photon. However, such states can be accessed by two photon (XUV + NIR) processes. In particular, the $3 s 3 p^{6} 4 d(28.3 \mathrm{eV})$ state lies $1.7 \mathrm{eV}$ above the $3 s 3 p^{6} 4 p$ state [23], which is within the spectral range of the few-cycle NIR pulse (1.3-2.1 eV) and is approximately equal to the central photon energy of the NIR laser pulse. When the two states are strongly coupled by the NIR laser, as is shown in Fig. 3(a) and 3(c), Rabi oscillations

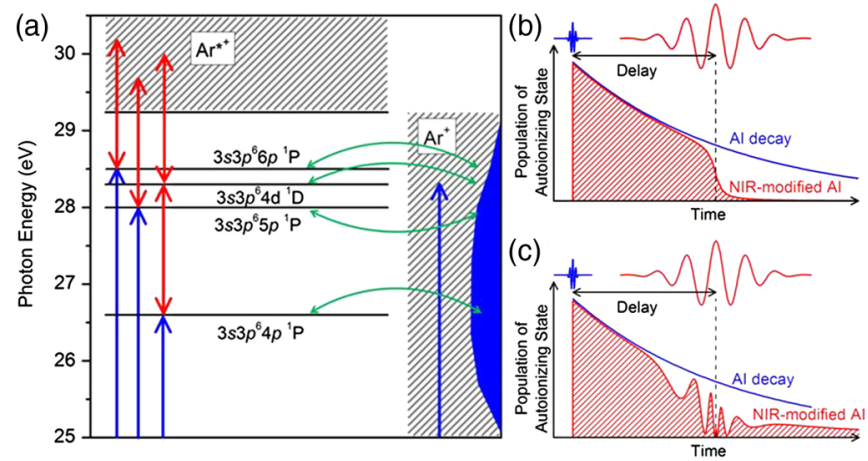

FIG. 3 (color online). (a) Schematic representation of argon autoionizing states exposed to the strong NIR laser field. The blue arrows indicate the attosecond XUV excitation of the ground state to the $3 s 3 p^{6} n p{ }^{1} P$ states as well as to the $\operatorname{Ar}^{+}\left(3 s^{2} 3 p^{5} \varepsilon l\right)$ continuum. The red arrows indicate the NIR laser coupling between the autoionizing states and the $\mathrm{Ar}^{*+}\left(3 s 3 p^{6} \varepsilon l\right)$ continuum or to $3 s 3 p^{6} n l$ autoionizing states. The configuration interaction (green arrows) couples all autoionizing states to the $\mathrm{Ar}^{+}$ continuum. (b) Autoionization decay modified by NIR laserinduced coupling to the $\operatorname{Ar}^{*+}\left(3 s 3 p^{6} \varepsilon l\right)$ continuum. Ionization by the NIR field truncates the autoionization decay, resulting in a shorter lifetime and a broader, shifted resonance peak. (c) Autoionization decay modified by NIR laser-induced coupling to $3 s 3 p^{6} n l$ autoionizing states. Rabi oscillation between the two states results in ac Stark-like splitting $[9,10]$. between the two states can cause ac Stark splitting of each coupled state $[9,10]$. In the case of pure bound states and resonant coupling, such splitting results in the symmetric Autler-Townes doublet [24]. However, the $3 s 3 p^{6} 4 p$ and $3 s 3 p^{6} 4 d$ states will autoionize by themselves without the participation of the NIR laser. The asymmetric Fano line shapes as well as the slight detuning of the laser central frequency from resonance complicate the inference and lead to the asymmetric splitting $[9,10]$.

Simulations of the laser-induced coupling of the $3 s 3 p^{6} 4 p$ and $3 s 3 p^{6} 4 d$ autoionizing states were performed based on the formalism in Refs. [9,10]. In our treatment, the $3 s 3 p^{6} 5 p$ and $3 s 3 p^{6} 6 p$ states as well as the $\mathrm{Ar}^{*+}$ $\left(3 s 3 p^{6} \varepsilon l\right)$ continuum are ignored, but coupling to the $\mathrm{Ar}^{+}\left(3 s^{2} 3 p^{5} \varepsilon l\right)$ continuum via configuration interaction is preserved. The wave function of the model system exposed to the combined laser fields is given by

$$
\begin{aligned}
|\psi(t)\rangle= & c_{g}(t) e^{-i E_{g} t}|g\rangle+c_{a}(t) e^{-i E_{a} t}|a\rangle+c_{b}(t) e^{-i E_{b} t}|b\rangle \\
& +\int d E_{a}^{c} c_{E_{a}^{c}}(t) e^{-i E_{a}^{c} t}\left|E_{a}^{c}\right\rangle \\
& +\int d E_{b}^{c} c_{E_{b}^{c}}(t) e^{-i E_{b}^{c} t}\left|E_{b}^{c}\right\rangle
\end{aligned}
$$

where $|g\rangle$ represents to the ground state of argon $\left|3 s^{2} 3 p^{6}\right\rangle$, and $|a\rangle,\left|E_{a}^{c}\right\rangle$ and $|b\rangle,\left|E_{b}^{c}\right\rangle$ represent the bound and continua parts of the two autoionizing states $\left|3 s 3 p^{6} 4 p\right\rangle$ and $\left|3 s 3 p^{6} 4 d\right\rangle$, respectively. Following Refs. [9,10], the coupled equations for the time-dependent amplitudes $c_{a}(t)$ and $c_{b}(t)$ were solved. The XUV and NIR laser pulse durations and intensities were chosen to be the same as in the experiment, and the energies, widths, and $q$ parameters of the autoionizing states were taken from the literature $\left(q_{a}=-0.2, q_{b}=2.43\right)[4,23]$. The dipole matrix elements $\langle g|z| a\rangle$ and $\langle a|z| b\rangle$ were calculated to be 0.027 and 1.54 a.u., respectively, using single particle wave functions calculated with an effective Coulomb potential [25]. The single-atom dipole radiation spectrum is given by

$$
\tilde{D}(\omega)=\left|\int_{-\infty}^{\infty} d t d(t) e^{i \omega t}\right|^{2}
$$

where $d(t)$ is the dipole matrix element

$$
\begin{aligned}
d(t) & =\langle\psi(t)|z| \psi(t)\rangle \\
& =2 \operatorname{Re}\left\{c_{a}(t) e^{-i\left(E_{a}-E_{g}\right) t}\langle g|z| a\rangle\left[1-\frac{i}{q_{a}}\right]\right\} .
\end{aligned}
$$

The simulated delay-dependent spectra are shown in Fig. 4(a) and 4(b) for NIR laser intensities of $5 \times 10^{11}$ and $1 \times 10^{12} \mathrm{~W} / \mathrm{cm}^{2}$, respectively. The features observed in the experiment, including the asymmetric splitting, broadening, and weakening of the Fano line shape, are reproduced very well by the model. 

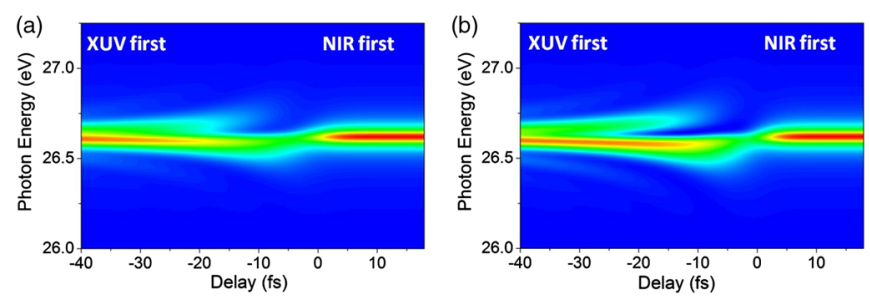

FIG. 4 (color online). Simulated dipole radiation spectrum of laser-induced coupling of the $3 s 3 p^{6} 4 p$ and $3 s 3 p^{6} 4 d$ autoionizing states. The XUV laser had a pulse duration of 140 as and intensity of $10^{10} \mathrm{~W} / \mathrm{cm}^{2}$. The NIR laser had a pulse duration of $8 \mathrm{fs}$ and intensity of (a) $5 \times 10^{11} \mathrm{~W} / \mathrm{cm}^{2}$ and (b) $10^{12} \mathrm{~W} / \mathrm{cm}^{2}$.

In conclusion, time-resolved autoionization of atoms with attosecond transient absorption spectroscopy was demonstrated for the first time. In the experiment, the autoionization process was initiated by an isolated attosecond pulse and modified by a NIR laser. By changing the delay between the two pulses, we have demonstrated control over the $E_{r}, \Gamma$, and $q$ parameters which characterize the autoionization process. Further control could be afforded by tuning the NIR laser central frequency with respect to the resonance. The capability of synchronizing an intense few-cycle NIR pulse and a 140 as XUV pulse on an argon gas target allows us to control the autoionization process with a lifetime of less than $10 \mathrm{fs}$. Coupling between such short-lived highly excited states has never before been studied in time-resolved experiments, as it requires laser pulses much shorter than the autoionization lifetime. Therefore, control of the autoionization process in argon clearly demonstrates that isolated attosecond pulses are crucial tools for studying electron correlation dynamics.

This work was supported by the U.S. Army Research Office under grant number W911NF-07-1-0475, the NSF, and by the Division of Chemical Sciences, Office of Basic Energy Sciences, Office of Energy Research, U.S. DOE.
*To whom correspondence should be addressed chang@phys.ksu.edu

[1] A. H. Zewail, J. Phys. Chem. A 104, 5660 (2000).

[2] P. B. Corkum and F. Krausz, Nature Phys. 3, 381 (2007).

[3] U. Fano, Phys. Rev. 124, 1866 (1961).

[4] R. P. Madden, D. L. Ederer, and K. Codling, Phys. Rev. 177, 136 (1969).

[5] X. J. Liu et al., Phys. Rev. Lett. 91, 193203 (2003).

[6] J. Faist et al., Nature (London) 390, 589 (1997).

[7] V. Madhaven et al., Science 280, 567 (1998).

[8] M. Hentschel et al., Nature (London) 414, 509 (2001).

[9] P. Lambropoulos and P. Zoller, Phys. Rev. A 24, 379 (1981).

[10] S. I. Themelis, P. Lambropoulos, and M. Meyer, J. Phys. B 37, 4281 (2004).

[11] M. Wickenhauser et al., Phys. Rev. Lett. 94, 023002 (2005).

[12] X. M. Tong and C.D. Lin, Phys. Rev. A 71, 033406 (2005).

[13] Z.X. Zhao and C. D. Lin, Phys. Rev. A 71, 060702(R) (2005).

[14] S. Gilbertson et al., International Quantum Electronics Conference (IQEC), Baltimore, Maryland May 31, 2009 (Optical Society of America, Washington, D.C., 2009).

[15] F. Krausz and M. Yu. Ivanov, Rev. Mod. Phys. 81, 163 (2009).

[16] L. Young et al., Phys. Rev. Lett. 97, 083601 (2006).

[17] Z. H. Loh et al., Phys. Rev. Lett. 98, 143601 (2007).

[18] S. Chen et al., Appl. Opt. 48, 5692 (2009).

[19] X. Feng et al., Phys. Rev. Lett. 103, 183901 (2009).

[20] M. Chini et al., Opt. Express 17, 21459 (2009).

[21] D. A. Mossessian et al., Nucl. Instrum. Methods Phys. Res., Sect. A 347, 244 (1994).

[22] N. B. Delone and V. P. Kranov, Phys. Usp. 42, 669 (1999).

[23] J. W. McConkey and J. A. Preston, J. Phys. B 6, L138 (1973).

[24] S.H. Autler and C.H. Townes, Phys. Rev. 100, 703 (1955).

[25] X. M. Tong and C. D. Lin, J. Phys. B 38, 2593 (2005). 